Wiegen- und Kinderlieder

Gesammelt durch die Brüder Grimm

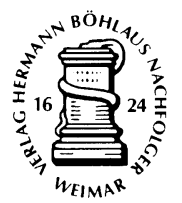




\title{
WIEGEN- UND KINDERLIEDER
}

\author{
Gesammelt durch die \\ Brüder Grimm
}

\section{Herausgegeben und kommentiert von Heinz Rölleke}

Mit zwölf zeitgenössischen Illustrationen und drei Faksimiles

\author{
1999 \\ Verlag Hermann Böhlaus Nachfolger Weimar
}


Die Deutsche Bibliothek - CIP-Einheitsaufnahme

Wiegen- und Kinderlieder / ges. durch die Brüder Grimm.

Hrsg. und kommentiert von Heinz Rölleke. -

Weimar : Verlag Hermann Böhlaus Nachfolger, 1999

ISBN 978-3-7400-1090-4

ISBN 978-3-7400-1090-4

ISBN 978-3-476-03328-4 (eBook)

DOI $10.1007 / 978-3-476-03328-4$

Dieses Werk einschließlich aller seiner Teile ist urheberrechtlich geschützt. Jede Verwertung außerhalb der engen Grenzen des Urheberrechtsgesetzes ist ohne Zustimmung des Verlages unzulässig und strafbar. Das gilt insbesondere für Vervielfältigungen, Übersetzungen, Mikroverfilmungen und die Einspeicherung und Verarbeitung in elektronischen Systemen.

(C) 1999 Springer-Verlag GmbH Deutschland

Ursprünglich erschienen bei Verlag Hermann Böhlaus Nachfolger Weimar 1999 


\title{
Inhalt
}

\author{
Vorwort \\ 1 \\ Wiegen- und Kinderlieder \\ 11
}

Abkürzungsverzeichnis

86

Kommentar

89

Alphabetisches Register

155

Bildnachweis

160 


\section{Vorwort}

Als Mitte 1808 ein Anhang zu den drei Bänden der Arnim/Brentanoschen Sammlung alter deutscher Lieder Des Knaben Wunderhorn erschien, und zwar ein etwas mehr als 100 Seiten umfassendes Heftchen mit dem schlichten Titel Kinderlieder, war das für die Zeitgenossen eine Art Sensation, für viele ganz gewiß eine Provokation. Die sich noch der Epoche der Aufklärung zuzählenden Rezensenten übergossen die liebevollen Bemühungen der jungen romantischen Dichter um die Sammlung und Bewahrung kindlicher Lieder und Spiele mit Spott und Hohn: Dies sei eine Zumutung für jeden vernünftigen Erwachsenen, in jedem Fall wertlos.

Angesichts dieses baren Unverständnisses muß man sich allerdings klarmachen, daß ein genuines Interesse an kindlichen Lebensformen und -äußerungen, ja an Wesen und Eigenheiten des Kindes überhaupt erst etwa eine Generation zuvor erstmals - und zwar mit entsprechenden Darlegungen Rousseaus - aufgekommen war. Die europäische Kunst hatte die Phänomene 'Kind und 'Kindlichkeit jahrhundertelang übergangen: Kinder wurden wie eine tabula rasa angesehen und waren in der Literatur sowie in der Bildenden Kunst nur insoweit von Interesse, als sie Züge des Erwachsenwerdens erkennen ließen. Die Darstellung des Kindes war demgemäß immer die eines kleinen, gegebenenfalls noch unfertigen Erwachsenen. Daß der junge Goethe 1773 in seinem Götz von Berlichingen den Sohn des Ritters tatsächlich als Kind auftreten und reden ließ, war eine absolute Novität.

Als Johann Gottfried Herder 1777/78 mit seiner für entsprechende Bemühungen bahnbrechenden Sammlung Volkslieder herauskam, schenkte er den Kinderliedern noch keinerlei Beachtung.

Es war erst die Epoche der Romantik, die mit ihrer 
Hochschätzung sowie alsbald forcierten Idealisierung der Kindlichkeit auch das Kinderlied, zunächst allerdings noch zögerlich, als volksliterarische Gattung zur Kenntnis nahm. Wie schwer man sich selbst in diesen Kreisen damit tat, erhellt aus einem Diktum August Wilhelm Schlegels, immerhin einer der Gründerväter der romantischen Bewegung: "Jede gute Wärterin soll ihr Kind unterhalten oder wenigstens beruhigen und einschläfern; leistet sie dies ... so ist weiter keine Forderung an sie zu machen. Wenn man aber die ganze Rumpelkammer wohlmeinender Albernheit ausräumt und für jeden Trödel ... Ehrerbietung begehrt, so wird in der Tat gescheiten Leuten allzu viel zugemutet. "Was sonst von der zeitgenössischen Kritik zu erwarten war und wie schief die Meßlatte war, an der man den Wert oder Unwert von Kinderliedern messen wollte, kann etwa die Persiflage des Spätaufklärers und Jugendfreundes Schillers Johann Christoph Friedrich Haug auf die Wunderhorn-Kinderlieder Brentanos verdeutlichen: "Was Mägde, Kinder und Ammen / Gefabelt und gestammelt, / Hat Er als olympische Flamme / Großmütig gütig zusammen / Für große Kinder gesammelt ... fad' und töricht, / Als Spreu und Kehricht, / Als puren Nonsens ... / Dies aufgeraffte Gemengsel ... / Einen Hohlkopf schalt ich / Den Sammler der Lieder für Kinder ... Der Schöpfer von >Troß, troß, trill / [gilt ihm mehr] als Homer und Vergil.»

Es war Wilhelm Grimm (1786-1859), der das so unsachgemäß verhöhnte Kinderlied »Troß, troß, trill" für Brentano aufgezeichnet hatte, so daß diese Kritik auch ihn direkt traf. Das hat ihn und seinen Bruder Jacob (1785-1863) jedoch keineswegs veranlaßt, die Beschäftigung mit den europäischen Kinderliedern in Form des Sammelns und Erläuterns aufzugeben: Das bezeugen entsprechende Veröffentlichungen und vor allem die Fülle der im Grimm-Nachlaß erhaltenen Zeugnisse.

Bevor davon die Rede ist, sei kurz referiert, wer und 
was die Brüder Grimm zu dieser für ihre Zeit noch so neuen und öffentlich scheel angesehenen Sammeltätigkeit veranlaßt hat.

Wie in der Vorgeschichte ihrer nachmals weltberühmten Sammlung der Kinder und Hausmärchen (1812/15) spielt auch für das Thema 'Kinderlieder Clemens Brentano die ausschlaggebende Rolle. Im Zuge der Vervollständigung seiner Volksliedsammeltätigkeit für Des Knaben Wunderhorn (der erste Band war Ende 1805 erschienen) schrieb er am 22. März 1806 an seinen Schwager Friedrich Carl von Savigny: "Haben Sie in Kassel keinen Freund, der sich dort auf der Bibliothek umtun könnte, ob keine alten Liedlein dort sind, und der mir dieselben kopieren könnte?« Savigny empfahl die Brüder Grimm, die bei ihm studiert hatten, und Jacob Grimm nahm im Juni 1806 den Auftrag an. Ähnlich wie bei der gleichzeitig begonnenen und ebenfalls durch Brentano angeregten Sammlung von Volksmärchen beschränkten die Brüder Grimm ihre Suche allerdings nicht auf die Schätze der Kasseler Bibliothek, sondern schenkten darüber hinaus besonders der mündlichen Tradition ihre Aufmerksamkeit. Anders als bei den Märchen kamen ihnen bei den Volksund Kinderliedern jedoch auch Erinnerungen an ihre Kindheit in den Sinn. So ergibt sich von Beginn an bei der Märchen- wie bei der Liedersammlung eine Gemengelage mit Materialien aus gedruckten wie aus mündlichen Quellen. Aus den von den seinerzeit knapp zwanzigjährigen Brüdern Grimm für die Wunderhorn-Bände 2 und 3 wie für die Kinderlieder beigetragenen Texten nahm Brentano nachweislich nicht weniger als 28 in seine Sammlung auf, doch ist mit einiger Sicherheit zu vermuten, daß gerade von den Kinderliedern bislang ungeklärter Provenienz noch eine ganze Reihe auf die Brüder Grimm zurückgeht. Schon zu Beginn ihrer Mitarbeit am Wunderhorn und erst recht nach dessen Abschluß sammelten die Brüder Grimm für ihre eignen Zwecke weiter - 
genauso waren sie mit den Märchen verfahren, die sie zunächst ausschließlich im Auftrag von sowie für Brentano gesammelt hatten und deren Sammlung sie für ihre eigenen Zwecke weiterführten, als der romantische Dichter seine entsprechenden Publikationspläne stillschweigend aufgegeben hatte. Nur: Ihre Märchensammlung bearbeiteten sie für den Druck und betreuten deren Veröffentlichung in insgesamt 17 Auflagen ihr Leben lang, während sie die ebenfalls zeit ihres Lebens gesammelten Volkslieder zwar teilweise für ihre wissenschaftlichen Veröffentlichungen heranzogen, die Textzeugnisse aber ansonsten zerstreuten, verschenkten oder in verschiedenenen Sammelmappen unredigiert (und unveröffentlicht) beließen.

Germanistik und Volkskunde sind erst relativ spät auf diese Schätze aufmerksam geworden - ausgewertet sind sie indes nur in Ansätzen. Dabei handelt es sich ausnahmslos um relativ frühe Zeugnisse, zuweilen um Erstbelege und Unikate. Erst seit 1975 wurden die Grimmschen Wunderhorn-Beiträge eruiert und vorgestellt, wobei auch einzelne Zeugnisse des hier erstmals insgesamt veröffentlichten Konvoluts herangezogen wurden (Des Knaben Wunderhorn, 6 Bde., hrsg. von H. Rölleke, Stuttgart u.a. 1975-78). Die große Ausgabe Brüder Grimm: Volkslieder (3 Bde., hrsg. von C. Oberfeld u.a., Marburg 1985-89) beschränkt sich auf den Marburger Grimm-Nachlaß, und die kleine Anthologie Brüder Grimm: Im Himmel steht ein Baum ... Volkslieder, Kinderlieder, Kinderzeichnungen (hrsg. von G. Seitz, München 1985) zieht lediglich zwei Zeugnisse aus dem Berliner Konvolut heran, ohne sie allerdings fehlerfrei wiederzugeben.

So handelt es sich bei der hier vorgelegten Veröffentlichung um den Erstdruck von Materialien, die seit dem Tod Jacob Grimms 1863 unter dem Konvoluttitel "Ammen, Wiegen, Kinderlieder " unsigliert und unpaginiert im Berliner Nachlaß aufbewahrt wurden (1975 von der Bibliotheks-Direktorin I. Stolzenberg, der für Auskünfte 
und Abdruckerlaubnis zu danken ist, dem "Kasten C« zugeschlagen). Es handelt sich um insgesamt 99 Blätter verschiedensten Formats und wechselnder Beschriftung. Die meisten Niederschriften stammen von Jacob und Wilhelm Grimm selbst; die erhaltenen Fremdhandschriften sind bislang in keinem Fall bestimmten Schreibern zuzuordnen. Der Zeitraum, in dem die Niederschriften dieser Kinderlieder zustande kamen, dürfte sich von 1806 bis nach 1829 erstrecken (s. Kommentar zu Anhang Nr. 1). Gingen die Brüder Grimm zu Beginn ihrer Sammeltätigkeit wohl noch einigermaßen systematisch vor wobei die überaus ergebnisreiche Auswertung des Schützeschen Idiotikons im Vordergrund stand -, so scheinen die später festgehaltenen Textzeugnisse eher sporadisch zustande gekommen $\mathrm{zu}$ sein. Man hatte wohl den Plan einer Ordnung, Kommentierung und Veröffentlichung - wenn er denn je bestanden haben sollte - spätestens seit 1818 (Abschluß der Deutschen Sagen) nicht weiter verfolgt. Immerhin wurden die Materialien z.B. in Wilhelm Grimms Aufsätzen über Kinderwesen und über Kinderglauben sowie in Jacob Grimms Deutscher Mythologie, aber auch noch im seit 1854 erscheinenden Deutschen Wörterbuch benutzt. Allein diese Tatsache macht deutlich, daß die Veröffentlichung dieser Grimmschen Kinderliedersammlung bislang ein Desiderat der Grimm-Forschung war.

In Übereinstimmung mit allen anderen volksliterarischen Sammlungen der Brüder Grimm kann auch über den Wiegen- und Kinderliedern eine Definition Jacob Grimms aus dem Jahr 1811 als Motto stehen: »Ihr gänzlich gelehrtes Ziel, das sich nichtsdestoweniger von jedermanns Ergötzlichkeit nicht entfernen wird, besteht im Zusammentragen von Materialien $\mathrm{zu}$ einer Geschichte deutscher Poesie." Auch diese Grimmsche Sammlung kann also - wenn auch notwendig in statu nascendi verblieben - literarhistorisches und gattungstheoretisches In- 
teresse der Germanistik wie der Volkskunde im allgemeinen beanspruchen sowie im besonderen der Volksund Kinderliedforschung mit neuen oder variierenden Materialien dienen. Daß dabei die von Jacob Grimm angesprochene "Ergötzlichkeit" des Lesers nicht zu kurz kommt, das versteht sich angesichts der oft zu Herzen gehenden Eigenart und der nicht selten unmittelbar ansprechenden Anmut dieses literarischen Genres von selbst.

Mit ihren Sammelbemühungen versuchten die Brüder Grimm stets, Alter und Geschichte der in Rede stehenden volksliterarischen Gattungen $\mathrm{zu}$ verdeutlichen oder $\mathrm{zu}$ bestimmen. Zeugnisse für Kinderlied und Kinderspiel finden sich indes im Mittelalter so gut wie gar nicht: Jacob Grimms Reklamation eines mittelhochdeutschen Gedichts als Wiegenlied (hier Anhang Nr.1; vgl. auch die Fehldeutung zu Nr.61) ist bezeichnenderweise irrig, und Wilhelm Grimm konnte in seinem Aufsatz über Kinderwesen nur wenige Belege aus der Kunstdichtung zur Diskussion stellen, ohne daß damit eine durchgängige mittelalterliche Tradition zu belegen wäre. Erst das Lied- und Spielverzeichnis, das Fischart 1575 in seiner Geschichtklitterung, wenn auch nur in Form von oft rätselhaften Stichwörtern, bietet, gestattet einige Vermutungen über den Sitz im Leben dieser Zeugnisse aus der Kinderwelt. Für das 17. Jahrhundert sind immerhin wenige Belege greifbar (vgl. z.B. Nr.2 und Nr.5), ehe dann zu Ende des 18. Jahrhunderts Aufzeichnungen und Veröffentlichungen in nennenswerterer Anzahl auftreten. Dabei ist auf eine Besonderheit hinzuweisen, die auch die früher so mangelhafte Überlieferungslage bezeugt: Wie Arnim und Brentano ausgerechnet den Almanachen Nicolais, die ausschließlich als Parodie und Verspottung der volksliterarischen Bemühungen Herders gedacht waren, einige ihrer wertvollsten Wunderhorn-Lieder verdankten (vgl. hier Nr.81), so entnahmen die Brüder Grimm der spöttischen 
Wunderhorn-Rezension der Hallischen Zeitung die anmutigen Verse vom "Goldsvögele" (Nr.64). Den eigentlichen Wert aber erhält die Grimmsche Sammlung natürlich durch die ausdrücklich als mündlich tradiert gekennzeichneten Zeugnisse, die durch etwa eine gleich große Anzahl solcher Texte ergänzt werden, deren Provenienz mit ziemlicher Sicherheit in der mündlichen Überlieferung der Zeit um 1800 anzusetzen ist.

Eine scharf umrissene Gattungsdefinition haben die Brüder Grimm hinsichtlich der Kinderlieder nicht gegeben, wie sie ja auch an ihre Märchen- und Sagensammlungen ohne theoretische Vorgaben herangingen. Für sie standen Praxis des Sammelns und Auswertung des Gesammelten von Anfang an und stets im Vordergrund. So mußte es natürlich zu einigen Grenzüberschreitungen und auch Irritationen kommen. Eine ganze Reihe von Volksliedern ist letztlich $\mathrm{zu}$ Unrecht $\mathrm{zu}$ den Kinderliedern geschlagen worden, teilweise durch Brentanos Vorgabe in den Wunderhorn-Kinderliedern veranlaßt (Nr.18, 19 usw.). Die Dichter, Forscher und Sammler waren sich eben um 1800 noch nicht im klaren, wodurch sich ein Kinderlied eigentlich definiert. Das Kompositum kann man theoretisch $\mathrm{ja}$ in vielfachem Sinn auffassen: ein Lied, das von Kindern handelt; ein Lied, das für Kinder gemacht ist; ein Lied, das von Kindern umgesungen oder gar erfunden wurde; oder schlichtweg ein Lied, das Kinder singen und /oder früher sangen. Damit ist die später zum wichtigsten Kriterium avancierte Frage der anonymen Herkunft noch nicht einmal angesprochen. Immerhin läßt sich bei den Grimmschen Lieder- wie bei den Märchensammlungen als bedeutendes Kriterium der Beweis oder die Vermutung ausmachen, daß diese Texte in der mündlichen Tradition lebendig waren oder noch sind. Ansonsten hebt Wilhelm Grimm in seinem Aufsatz über Kinderwesen darüber hinaus auf den Aspekt der kindlichen Auffassungen und Formulierungen $\mathrm{ab}$, wenn er zum Thema »Kinderlieder« aus- 
führt: "Sie enthalten einen einzelnen poetischen Gedanken, ein Bild, ein Gleichnis, oft ohne einen weiteren Zusammenhang. Das Kind blickt mit seinen reinen Augen umher, ein Vogel fliegt vorbei, ein Käferchen setzt sich auf seine Hand, ein Blümchen liegt neben ihm im Gras, ein armes Mädchen sitzt unter einem Baum und weint, das wird ganz unschuldig und kindlich vorgestellt, und darin liegt der eigene Reiz dieser Lieder." Bei dieser idealtypischen Sicht kommt natürlich nichts von den in diesem Genre besonders interessanten Produktions-, Distributions- und Rezeptionsweisen in den Blick - dafür war allerdings auch seinerzeit weder eine hinreichende Materialbasis noch eine zureichende literaturtheoretische Kenntnis gegeben. Daß die Brüder Grimm dazu indes einigen Grund gelegt und überdies lange Zeit hindurch mit spürbarer Freude den Spuren des Kinderlieds und Kinderspiels nachgegangen sind, dafür sollte man ihnen Dank wissen, indem man ihren durch die sogenannte spezifisch Grimmsche >Andacht zum (nur scheinbar) Unbedeutenden ' geprägten Arbeiten Beachtung schenkt und Respekt zollt, wie sie sich in der vorliegenden Edition, die ausnahmslos buchstabengetreu verfährt, zeigen möchten.

\section{Text und Kommentierungsverfahren}

Die Brüder Grimm haben die Materialien ihrer Sammelmappe absolut nicht geordnet. Wollte man die Texte in der völlig zufälligen Reihenfolge der Niederschriften wiedergeben, so würde sich notwendig ein Bild der Verwirrung bieten, wo etwa einerseits thematisch und inhaltlich $\mathrm{Zu}$ sammengehöriges weit voneinander entfernt, andererseits entsprechend Disparates nebeneinander stünde. Da eine Anordnung nach Quellen und Beiträgern angesichts der Materiallage nicht angebracht erscheint, hat sich ein Aufbau nach Themen empfohlen, so daß ähnlich dem Ver- 
fahren in Brentanos Kinderliedern etwa dem Heranwachsen des Kindes gefolgt wird: von den Wiegenliedern, zu den Spiel- und ABC-Liedern bis hin zu Themen des Erwachsenwerdens (in der Kindermund-Artikulation) und zu den fremdsprachigen Texten. Daß dabei nicht immer scharf umrissene Grenzen gezogen werden konnten und manche Zuordnung zweifelhaft bleiben muß, liegt in der Natur der Sache.

Die Durchnumerierung entspricht der vom Editor getroffenen Anordnung. Alle handschriftlichen Anmerkungen zur Herkunft oder zur Erklärung der Texte sind am Beginn der jeweiligen Kommentare in entsprechend markierter Schriftart (buchstabengetreu) wiedergegeben. Einfügungen des Herausgebers sind durch spitze, Streichungen in der Handschrift durch eckige Klammern markiert; in der Handschrift unterstrichene Texte erscheinen kursiv.

Die Kommentare wollen einer Interpretation und Auswertung der hier erstmals veröffentlichten Texte nicht vorgreifen; sie beschränken sich auf eruierbare Quellen- und relevante Parallelnachweise sowie einige Erläuterungen.

Die abschließenden Literaturangaben nennen die Standardwerke derVolks- und Kinderliedsammlungen, wo sich in der Regel weiterfuhrende Kommentare und Literaturhinweise finden. Darüber hinaus wurde die Sammlung Lewalters herangezogen, weil sie ähnlich wie die Grimmsche Sammlung weitgehend auf mündlicher Überlieferung in Kassel basiert - wenn auch einhundert Jahre nach Grimm. Eine in Anthologien und in der Forschung bislang so gut wie gar nicht ausgewertete anonyme Veröffentlichung, die 1836 in Bremen erschien (Kinder- und Ammen-Reime in plattdeutscher Mundart), ist wegen der oft erstaunlichen Übereinstimmungen mit und wegen der Parallelen zu den Grimmschen Materialien ebenfalls vollständig in den Literaturangaben berücksichtigt.

Neuss, den 28. Februar 1999

Heinz Rölleke 